\title{
INTERSEÇÃO DE SABERES EM MÍdIAS SOCIAIS PARA EDUCAÇÃO EM SAÚDE NA PANDEMIA DE COVID-19
}

KNOWLEDGE INTERSECTION IN SOCIAL MEDIA FOR HEALTH EDUCATION IN THE COVID-19 PANDEMIC

INTERSECCIÓN DE SABERES EN REDES SOCIALES PARA EDUCACIÓN EN SALUD DURANTE LA PANDEMIA DE COVID-19

Márcia Maria Santos da Silva

Kyaya Gomes de Carvalho 2

Iandra Karla da Silva Cavalcante ${ }^{3}$

Maria José Galdino Saraiva ${ }^{4}$

Roselane da Conceição Lomeo ${ }^{5}$

Polyanne Rodrigues Vasconcelos 6

Palavras-chave:

Educação em Saúde; Educação Interprofissional; Mídias Sociais; Pandemia; Coronavírus.

Keywords: Health Education; Interprofessional Education; Social Media; Pandemic; Coronavirus.

Palabras clave: Educación en Salud; Educación Interprofesional; Redes Sociales: Pandemia; Coronavirus.

Submetido: 26 de Set. de 2019

Aprovado: 11 de Nov. de 2020 Márcia Maria Santos da Silva Escola de Saúde Pública Visconde de

Saboia

R. Santana do Acaraú, 280 Pedro Mendes Carneiro - Sobral, CE CEP: 62030-712

E-mail:marciamss@yahoo.com.br
Autor(a) para Correspondência:

\section{RESUMO}

A pandemia de Coronavirus Disease 2019 (COVID-19) vem gerando demandas complexas e inesperadas para o Sistema Único de Saúde (SUS), as quais suscitaram ações emergenciais a curto, médio e longo prazo. Uma importante estratégia no combate à doença tem sido a educação em saúde, que pode ser efetivada em espaços formais e não formais, mas precisa estar contextualizada e contribuir com a transformação de modos de vida. Para que a população tenha acesso a informações dos diversos campos de saberes, o uso das mídias sociais é um recurso significativo, pois amplia o raio de alcance e possibilita a diversidade de orientações. A partir dessas premissas foi implementado o perfil do Programa de Educação pelo Trabalho para a Saúde (PET-Saúde) Interprofissionalidade de Sobral-CE na rede social Instagram para disseminar conteúdos sobre a promoção da saúde diante da pandemia de COVID-19. Assim, este artigo tem por objetivo compreender a interprofissionalidade na educação em saúde por meio das mídias sociais na pandemia de COVID-19. A experiência se centrou no trabalho interprofissional e contribuiu com a conscientização sobre os meios de prevenção da COVID-19 e promoção de hábitos mais saudáveis. As redes sociais se mostram ferramentas importantes para a educação em saúde, pois instigam reflexões sobre as possibilidades de adotar ações de cuidado promotoras da saúde e da qualidade de vida, de modo colaborativo e dialógico.

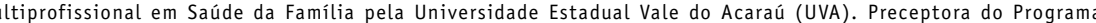
de Educação pelo Trabalho para a Saúde (PET-Saúde) Interprofissionalidade de Sobral-CE. E-mail: marciamss@ yahoo.com.br ORCID: https://orcid.org/0000-0001-6534-8196

2. Graduanda em Psicologia pela Universidade Federal do Ceará (UFC). Monitora do PET-Saúde Interprofissionalidade de Sobral. E-mail: kyaya_carvallho@hotmail.com ORCID: https://orcid.org/0000-0002-4286-5358

3. Graduanda em Educação Física pela UVA. Monitora do PET-Saúde Interprofissionalidade de Sobral. E-mail: iandrak21@hotmail.com 0RCID: https://orcid.org/0000-0001-5085-1098

4. Mestre em Ensino na Saúde pela UECE. Especialista em Formação Integrada Multiprofissional em Educação Permanente em Saúde pela Universidade Federal do Rio Grande do Sul (UFRGS) Preceptora do PET-Saúde Interprofissionalidade de Sobral.E-mail: mariajosegaaldinosaraiva@gmail.com 0RCID: https://orcid.org/00000001-6006-9091

5. Doutora em Educação pela Universidade de Aveiro (Portugal). Coordenadora de grupo tutorial do PET-Saúde Interprofissionalidade de Sobral. E-mail: lomeoroselane@yahoo.com.br 0RCID: https://orcid.org/0000-0002$\underline{5290-2749}$

6. Graduanda em Enfermagem pela UVA. E-mail: polyannerod@gmail.com ORCID: https://orcid.org/0000-0002 9929-7267

Certificação de redação científica: E.L.Freire Editora. Edição de texto: Evandro L. Freire. Revisão de provas: Texto definitivo lido e validado pelas autoras. 


\section{ABSTRACT}

The Coronavirus Disease 2019 (COVID-19) pandemic has been generating complex and unexpected needs for the Brazilian National Health System (Sistema Único de Saúde [SUS]), which have triggered emergency actions in the short-, medium-, and long term. A major strategy in fighting the disease has been health education, which can be put into practice in formal and non-formal spaces, but it needs to be contextualized and contribute to change ways of life. For the population to have access to information from various fields of knowledge, using the social media is a significant resource, as it broadens the reach and enables the diversity of guidelines. Based on these premises, the Instagram profile of the Program Education through Work for Health (Programa de Educação pelo Trabalho para a Saúde [PET-Saúde]) Interprofessionality in Sobral, Ceará, Brazil, has been created to disseminate content on health promotion in face of the COVID-19 pandemic. Thus, this article aims to grasp interprofessionality in health education through the social media in the COVID-19 pandemic. The experience focused on interprofessional work and it has contributed to raising awareness about the means of preventing COVID-19 and promoting healthier habits. The social media are important tools for health education, as they instigate reflections on the possibilities of taking appropriate actions that promote health and quality of life, in a collaborative and dialogical way.

\section{RESUMEN}

La pandemia de Coronavirus Disease 2019 (COVID-19) ha venido generando necesidades complejas e inesperadas para el Sistema Único de Salud de Brasil (Sistema Único de Saúde [SUS]), que han desencadenado acciones de emergencia en el corto, mediano y largo plazo. Una estrategia importante en la lucha contra la enfermedad ha sido la educación en salud, que se puede poner en práctica en espacios formales y no formales, pero necesita ser contextualizada y contribuir a cambiar formas de vida. Para que la población tenga acceso a información de diversos campos de saberes, el uso de las redes sociales es un recurso significativo, ya que amplía el alcance y posibilita la diversidad de orientaciones. Con base en estas premisas, se ha creado el perfil de Instagram del Programa de Educación a través del Trabajo para la Salud (Programa de Educação pelo Trabalho para a Saúde [PETSaúde]) Interprofesionalidad en Sobral, Ceará, Brasil, para difundir contenidos acerca de promoción de la salud durante la pandemia de COVID-19. Así, este artículo tiene como objetivo comprender la interprofesionalidad en la educación en salud a través de las redes sociales durante la pandemia de COVID-19. La experiencia se centró en el trabajo interprofesional y ha contribuido a concienciar acerca de los medios para prevenir la COVID-19 y promover hábitos más saludables. Las redes sociales son herramientas importantes para la educación en salud, ya que promueven reflexiones acerca de las posibilidades de tomar acciones adecuadas que promuevan la salud y la calidad de vida, de manera colaborativa y dialógica.

\section{INTRODUÇÃ O}

A sociedade pós-moderna não esperava ver seu constante fluxo de desenvolvimento bruscamente interrompido por uma pandemia. Não obstante, o ano de 2020 teve início com o anúncio, por parte da Organização Mundial da Saúde (OMS), da propagação da Coronavirus Disease 2019 (COVID-19), doença causada pelo novo coronavírus, que configurou uma emergência de saúde pública de importância internacional (ESPII) - o mais alto nível de alerta da OMS -, sendo posteriormente caracterizada como uma pandemia ${ }^{1}$.

Segundo o Ministério da Saúde $(M S)^{2}$, o novo coronavírus foi descoberto em 31/12/2019, após casos registrados na China, mais especificamente na cidade de Wuhan. A COVID-19 é causada pelo SARSCoV-2, que pertence à família dos coronavírus, que causa infecções respiratórias. Alguns sintomas dessa doença são tosse, febre, coriza, dificuldade para respirar e dor de garganta, podendo variar desde um resfriado simples até uma pneumonia severa.

No Brasil, o primeiro caso de COVID-19 foi registrado em 26/02/2020, em São Paulo-SP. Conforme dados do MS, a curva de contágio teve um crescimento progressivo e, em 21/06/2020, dados nacionais apontaram a marca de 50.617 óbitos pela doença. De 26/02/2020 a 19/09/2020 foram confirmados 4.717 .991 casos e 141.406 óbitos, colocando o país no segundo lugar mundial em número de óbitos registrados por COVID-19 - atrás apenas dos Estados Unidos da América (EUA) ${ }^{3}$.

0 Ceará, em período similar, também apresentou dados preocupantes e adotou medidas mais restritivas em função da doença. 0 Município de Sobral, localizado no norte do estado, foi marcado 
por um cenário epidemiológico crítico no período de março a outubro de 2020 (Figura 1).

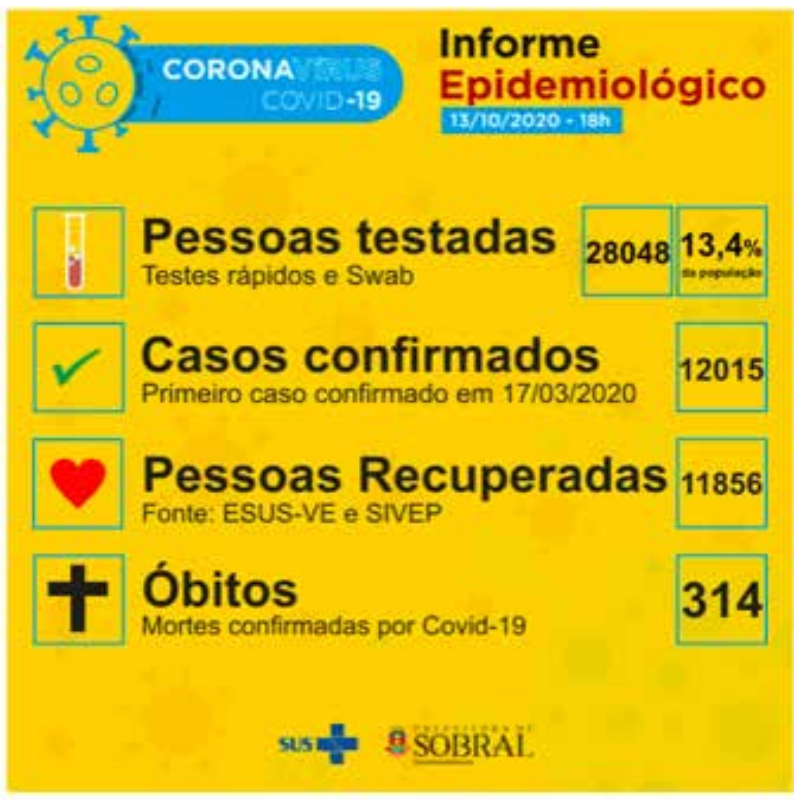

Figura 1 - Panorama de Casos de COVID-19. Sobral, 2020.

Fonte: Acervo pessoal das autoras.

$\mathrm{Na}$ condução das ações voltadas à pandemia, a gestão municipal adotou um plano de contingência para a COVID-19 e definiu diversas estratégias de isolamento social, uso obrigatório de máscara de proteção e restrição das vias de acesso a Sobral.

De acordo com a $0 \mathrm{MS}^{1}$, a maioria dos pacientes com COVID-19 (cerca de $80 \%$ ) pode ser assintomática e cerca de $20 \%$ dos casos podem requerer atendimento hospitalar por envolver dificuldade respiratória dentre esses casos, cerca de $5 \%$ podem necessitar de suporte ventilatório para o tratamento de insuficiência respiratória. Por se tratar de uma doença respiratória de fácil contágio, uma das medidas mais efetivas de prevenção e contenção da pandemia é o isolamento social, por ainda não existirem vacinas e medicamentos comprovadamente eficazes para curá-la ${ }^{1}$.

Uma das principais ferramentas no combate à COVID-19 tem sido a educação em saúde, com o compartilhamento de informações sobre as medidas de prevenção e tratamento dessa doença, considerando a fragilidade no que tange às alternativas medicamentosas eficazes para combatê-la. Um estudo ${ }^{4}$ destaca que a educação em saúde desenvolve uma interface entre a educação e a saúde com base no pensamento crítico sobre a realidade, como uma forma de transformação das condições objetivas que levem à promoção da saúde como direito de todos a partir de sua autonomia e emancipação históricosocial.

A educação em saúde envolve ações para orientar a população acerca de práticas saudáveis e responsabilidade em saúde, com a participação de diferentes categorias da saúde, além de propiciar a atuação interprofissional. A prática interprofissional em saúde se caracteriza pelo fato de duas ou mais profissões do setor saúde aprenderem com, para e sobre a outra, de modo a desenvolver uma colaboração mediada por um processo de aprendizagem compartilhada voltado a aprimorar a qualidade dos serviços prestados ${ }^{5}$.

No contexto atual, no qual as rotinas vêm sendo retomadas gradativamente, com a consequente saída do isolamento social, para que as pessoas tenham acesso a informações dos diversos campos de saberes, o uso das mídias sociais tem se mostrado um recurso vital, pois amplia o raio de alcance e proporciona uma diversidade de orientações com foco na promoção e proteção da saúde. Um estudo ${ }^{6}$ aponta que as mídias e as plataformas virtuais não são meras mediadoras ou espaços isolados do cotidiano, elas integram a vida e constituem importantes canais de comunicação que participam ativamente das ações humanas diárias. Em termos de educação em saúde em um cenário completamente novo, onde o contato físico precisa ser limitado ao máximo, encontramos nesses meios de comunicação a possibilidade de transmitir conhecimentos e informações sobre práticas de autocuidado que alcancem o maior número possível de pessoas.

A criação de um perfil na rede social Instagram foi uma iniciativa do Programa de Educação pelo Trabalho para a Saúde (PET-Saúde) Interprofissionalidade de Sobral-CE, implantado no município em 2019 pela Secretaria da Saúde e pela Escola de Saúde Pública Visconde de Saboia (ESP-VS), em parceria com a Universidade Federal do Ceará (UFC) e a Universidade Estadual Vale do Acaraú (UVA).

\section{Uma das principais ferramentas no combate à COVID-19 tem sido a educação em saúde...}


Assim, este artigo tem por objetivo compreender a interprofissionalidade na educação em saúde por meio das mídias sociais na pandemia de COVID-19. Busca-se compartilhar a experiência interprofissional de educação em saúde mediada pelas mídias sociais, com vistas a estimular práticas de autocuidado e empoderamento da população no contexto da pandemia de COVID-19.

\section{METODOLOGIA}

Trata-se de relato de experiência de monitores, preceptores e tutores do PET-Saúde Interprofissionalidade de Sobral, partindo da criação e manutenção de um perfil no Instagram, voltado ao enfrentamento da pandemia de COVID-19 em nível local. A proposta teve início após o Decreto Municipal n. 2.376/2020 , que instaurou o isolamento social como importante medida para evitar a disseminação do novo coronavírus.

A adoção do isolamento social provocou significativas mudanças nas rotinas locais, inclusive nos serviços de saúde e educação, nos quais diversas atividades presenciais foram suspensas ou cederam espaço para o formato virtual. Diante da inquietude no cenário pandêmico, o grupo tutorial do PETSaúde Interprofissionalidade de Sobral realizou um encontro virtual para que todos pudessem propor alternativas de contribuição. Foram consideradas as restrições das autoridades sanitárias no que concerne às atividades que reúnem pessoas, bem como as orientações de permanência de toda a população em seus domicílios, salvo necessidades de acesso a serviços de saúde essenciais, aquisição de gêneros alimentícios e medicamentos. Todavia, atentou-se ao potencial do grupo tutorial, sua multiplicidade de saberes, à predisposição ao trabalho colaborativo e ao imperativo ético posto aos profissionais e estudantes do setor saúde diante da pandemia.

observando o aumento do uso de redes sociais, a possibilidade de disseminação de materiais educativos sob a forma de imagens e vídeos e a viabilidade de interação com o público, o grupo tutorial reconheceu o potencial do uso desses canais de comunicação para orientar a população quanto aos cuidados necessários para a prevenção do novo coronavírus. Outrossim, tal iniciativa se mostrou um veículo indispensável à formação e ao trabalho colaborativo entre os membros do grupo.

0 planejamento da atividade possibilitou a definição de diretrizes que nortearam a atuação

\section{...a educação em saúde desenvolve uma inter face entre a educação e a saúde com base no pensamento crítico sobre a realidade...}

de preceptores e monitores do PET-Saúde Interprofissionalidade de Sobral, dentre elas: a) a responsabilidade na identificação de conteúdo em fontes confiáveis (sites oficiais relacionados à saúde pública); b) o cuidado com a seleção de materiais pertinentes e de fácil compreensão; c) o respeito à diversidade de conteúdos, conforme indicação de todos os membros do grupo; d) a atenção quanto aos conteúdos postados, guardando coerência e evitando repetição de informações; e e) o compromisso com o envio do material na data programada.

Em seguida, definiu-se o Instagram como canal de comunicação de escolha, considerando suas possibilidades de compartilhamento de informações, suas condições de acesso livre e sua boa aceitação pública. Foi criado um fluxo de comunicação e de programação das postagens, seguindo um cronograma onde todos podem compartilhar saberes a partir de sua formação, gerando uma diversidade de conteúdos de várias categorias profissionais do setor saúde, como Medicina, Psicologia, Enfermagem, Educação Física e Odontologia. As postagens, sob a forma de imagens e vídeos, contemplam: a) dados epidemiológicos; b) prevenção do novo coronavírus; c) cuidados em saúde mental; d) cursos relativos ao setor saúde; e) prática de atividades físicas; f) hábitos alimentares saudáveis; g) alertas sobre fake news etc.

Prezando pela segurança das informações compartilhadas, realiza-se um cuidadoso trabalho de seleção das postagens por parte de todos os membros do grupo, verificando fontes seguras e comprometidas com a veracidade das informações. Ademais, para assegurar a constância das postagens foram designados dois monitores para 0 compartilhamento diário dos conteúdos fornecidos pelos membros do PET-Saúde Interprofissionalidade de Sobral. Essa definição se mostrou importante para proporcionar maior organicidade ao fluxo de postagens e resguardou o acesso às informações do perfil criado. 
Para verificar o alcance e a relevância dos conteúdos compartilhados no Instagram, além de analisar como a interprofissionalidade contribui com a educação em saúde durante o isolamento social, assegurou-se que a experiência constaria na pauta das reuniões virtuais do grupo tutorial.

\section{RESULTADOS E DISCUSSÃO}

Inicialmente, a experiência possibilitou a identificação do uso das mídias sociais no cotidiano de milhares de pessoas, dentre elas diversos profissionais do setor saúde que buscam ferramentas acessíveis para a prevenção e o enfrentamento da COVID-19. Tal constatação decorre do elevado número de internautas que interagem com os conteúdos postados em perfis públicos que abordam a temática no Instagram.

A criação do perfil PET-Saúde Interprofissionalidade Sobral no Instagram se mostra uma estratégia interprofissional de educação em saúde fundamental no período pandêmico. Tal canal informativo é alimentado diariamente com base no trabalho colaborativo de seus membros, que apresentam variadas formações acadêmicas, evidenciando proatividade, compromisso e respeito com a identificação e o compartilhamento de conteúdos relevantes à promoção da saúde e ao combate do novo coronavírus.

A iniciativa tem constatado como as práticas interprofissionais se mostram importantes para - cuidado integral da população, contemplando tanto a promoção da saúde quanto a prevenção de doenças. Tais práticas podem contribuir com a difusão do cuidado integral voltado ao paciente, isento de hierarquias de saber/poder profissional, onde a mutualidade e a colaboração tornam o fazer saúde fluido e holístico. Um estudo ${ }^{8}$ aponta que a interprofissionalidade se expressa pelo trabalho de diferentes profissionais do setor saúde que atuam a partir de um interesse comum, apresentando características marcantes, como aumentar a segurança da assistência, reduzindo riscos e danos, reforçando as ações de prevenção de doenças e promoção da saúde e aprimorando a integralidade da atenção à saúde.

A busca reiterada dessa abordagem centrada na integralidade da atenção à saúde considera aspectos como as alterações no perfil demográfico e epidemiológico da população brasileira e na complexidade da rede de atenção à saúde como um todo. Ao refletir sobre a interprofissionalidade, um estudo ${ }^{9}$ considera que os desafios do trabalho no âmbito do SUS remetem à necessidade de práticas integradas e colaborativas.

Esta experiência no Instagram fomentou 0 trabalho colaborativo entre os participantes percebido como importante ferramenta para que as práticas públicas no setor saúde proporcionem diálogo e abertura entre os profissionais, reverberando cuidados mais assertivos e integrados. 0 trabalho no âmbito do SUS reúne profissionais de diferentes áreas do saber e requer sua ativa participação. É imprescindível que tais profissionais desenvolvam práticas integradas e colaborativas com propósitos em comum, balizados em princípios como a universalidade do acesso ao SUS e a integralidade dos serviços de saúde. Um estudo ${ }^{10}$ indica que as práticas colaborativas se materializam quando profissionais de diferentes áreas atuam com base na integralidade da saúde em caráter permanente, reconhecendo a centralidade do usuário dos serviços de saúde, além dos relevantes papéis assumidos por suas famílias e sua comunidade.

No contexto da pandemia de COVID-19, a reorganização dos serviços de saúde municipais de Sobral evidenciou para os membros do grupo tutorial que as práticas interprofissionais são necessárias em quaisquer contextos, embora nem sempre seja possivel que se efetivem presencialmente. Ao mesmo tempo, o grupo constatou que as mídias sociais ampliam seu raio de atuação, sensibilizando a população quanto às medidas necessárias ao autocuidado e ao tratamento.

Os serviços de saúde pública se deparam com o desafio de educar a população seguindo as normativas definidas pela OMS, ao passo que se disseminam campanhas informativas acerca do combate e do combate à propagação do novo coronavírus, com a oportunidade de promover o encontro virtual entre os profissionais da saúde e a população $0^{11}$. Em meio às

\section{Esta experiência no Instagram fomentou o trabalho colaborativo entre os participantes...}


inúmeras informações disponíveis, a identificação de material educativo para compartilhar via Instagram demandou atenção e responsabilidade por parte de todos os membros do grupo quanto às fontes dos conteúdos.

A orientação comum aos participantes da experiência para identificar conteúdos em páginas oficiais expressa um compromisso ético que envolve o compartilhamento de informações precisas, reduz o erro e a incerteza decorrentes do excesso das chamadas fakes news. Estas representam um sério risco à saúde pública, pois a desinformação compromete o comportamento das pessoas, expondo-as a grandes riscos de contágio por falta de mobilização populacional no enfrentamento da COVID-19. Um estudo ${ }^{12}$ ratifica que as notícias falsas afetam de modo muito rápido e negativo um grande número de pessoas.

Um dos principais aspectos tanto do excesso quanto à qualidade de informações, muito presente neste período pandêmico, diz respeito à chamada infoxicação. Esse termo é um neologismo cunhado pelo físico Alfons Cornellá na década de 1990 e remete à relação entre informação e intoxicação. Um estudo ${ }^{13}$ explica que a infoxicação expressa a dificuldade para digerir o excesso de conteúdos e aferir a qualidade, a veracidade e a relevância das informações. A infoxicação se desenvolve em 3 fases: a) busca (excesso de conteúdos); b) seleção (fragilidade na comprovação da veracidade); e c) processamento (leitura fragmentada e superficial) as quais coadunam com a disseminação de conteúdos sem análise crítica por parte dos internautas. Como desdobramentos, a infoxicação pode gerar sintomas como irritabilidade, insônia, instabilidade de humor, ansiedade e estresse. Constata-se, assim, a importância da adoção de políticas públicas e legislações que normatizem aspectos relativos a segurança, privacidade e diretrizes de uso das tecnologias digitais.

Não obstante, as tecnologias da informação e da comunicação (TIC) se mostram importantes ferramentas em processos educativos - sejam elas formais ou não. A opção pelo uso das mídias sociais como estratégia para promover a educação em saúde, contemplando conteúdos pertinentes e confiáveis, considerou o pressuposto de que elas induzem a busca de autonomia por parte dos sujeitos diante do cuidado consigo e com o outro no processo saúde-doença. Um estudo 14:32 refere que "a Educação em Saúde é um processo essencialmente ativo que

\section{...as tecnologias da informação e da comunicação (TIC) se mostram importantes ferramentas em processos educativos...}

envolve uma mudança no modo de pensar, sentir e agir dos indivíduos".

As ações de educação em saúde envolvem 3 segmentos: a) profissionais do setor saúde; b) gestores do setor saúde; e c) usuários dos serviços de saúde e população brasileira - que estão fortemente presentes no trabalho em saúde. Essas ações podem ser efetivadas em espaços formais e não formais e devem ser contextualizadas, contemplando as diversas maneiras de democratizar o acesso a informações, impulsionar a produção de saberes e, por conseguinte, transformar os modos de vida ${ }^{15}$.

Vale observar que são variados os conteúdos contemplados na experiência da educação em saúde mediada por tecnologias digitais, correlacionados com uma perspectiva de saúde que vai além da pandemia de COVID-19 e estimula o encontro de diferentes saberes e a participação de diversas categorias profissionais. Práticas integradas ensejam a experiência interprofissional, como se observou na participação dos membros do PETSaúde Interprofissionalidade de Sobral na criação e manutenção do perfil no Instagram.

Dentre as evidências relativas à experiência, destacam-se os acessos à conta criada pelo grupo tutorial por perfis públicos e privados, incluindo profissionais e instituições do setor saúde, bem como a interação dos seguidores em postagens e quizzes e enquetes realizadas por meio do perfil. Também merece ênfase a participação ativa de todos os membros no processo de divulgação de informações - desde a busca de materiais até a produção de conteúdos autênticos e dialógicos.

A experiência trouxe importantes contribuições, nos campos teórico e prático, para os participantes. De imediato, destaca-se o aprofundamento conceitual e metodológico acerca da interprofissionalidade em saúde e das práticas colaborativas, o que contribuiu com o refinamento da aprendizagem dos membros do grupo tutorial. Também se ressalta a maior compreensão 
sobre o fazer profissional de todos aqueles que compõem o grupo, possibilitando vislumbrar alternativas de ações interprofissionais. Outro aprendizado corresponde à compreensão da educação em saúde como difusora de saberes, agregando aspectos culturais e sociais às orientações sobre práticas saudáveis. Nesse sentido, observa-se que a vivência favoreceu a articulação de conhecimentos advindos do PET-Saúde Interprofissionalidade de Sobral como fatores basilares para as intervenções e as orientações em saúde - inclusive no contexto da pandemia de COVID-19.

\section{CONCLUSÃO}

A pandemia de COVID-19 vem gerando demandas complexas e inesperadas para o SUS, as quais suscitaram ações emergenciais a curto, médio e longo prazo. As orientações da OMS e os achados científicos a partir de experiências em outros países se mostraram fundamentais para direcionar gestores e profissionais da saúde e a população brasileira neste momento crítico. Não obstante, a propagação do novo coronavírus e o comportamento descompromissado de diversos grupos acendeu um alerta quanto à necessidade de estratégias de enfrentamento da COVID-19.

Além de intensificar as ações de atenção à saúde voltadas a pessoas adoecidas, o atual cenário requer atitudes de corresponsabilidade entre os profissionais da saúde e a população quanto às medidas de prevenção dessa doença. Nesse sentido, a educação em saúde se mostra uma importante estratégia para orientar acerca de atitudes possíveis e necessárias. Esse pressuposto foi o fio condutor da experiência interprofissional de educação em saúde mediada por tecnologias digitais voltada a disseminar ações promotoras da saúde nas comunidades.

0 crescimento do uso das mídias sociais como disseminadoras de informações constitui um terreno fértil para semear as ações de educação em saúde, ainda que não cheguem de modo massivo àquelas parcelas mais empobrecidas da população. Tal contribuição se mostra significativa sob a perspectiva de promover a conscientização das pessoas quanto à necessidade de adotar hábitos mais saudáveis, sobretudo tendo como base os diversos olhares profissionais que se entrelaçam no PETSaúde Interprofissionalidade de Sobral.

$\mathrm{Na}$ experiência em tela, conviveu-se com o desafio de superar o enfoque uniprofissional e a perspectiva

\section{...Práticas \\ integradas ensejam a experiência interprofissional...}

reducionista nas orientações postadas no Instagram. Ao final, constatou-se que o atual momento de crise propicia importantes aprendizagens sobre práticas colaborativas e que a interprofissionalidade cresce a olhos vistos nas ações de educação em saúde. 0 cotidiano dessa experiência viabilizou a interseção de saberes, com todas as suas possibilidades, seus dilemas e seus desafios, fomentando novas percepções da interprofissionalidade no setor saúde.

\section{CONTRIBUIÇÃO DAS AUTORAS}

Márcia Maria Santos da Silva contribuiu com a realização da pesquisa, o delineamento do estudo e a redação e revisão crítica do manuscrito. Kyaya Gomes de Carvalho, Iandra Karla da Silva Cavalcante e Maria José Galdino Saraiva contribuíram com a realização da pesquisa e a redação do manuscrito. Roselane da Conceição Lomeo e Polyanne Rodrigues Vasconcelos contribuíram com a realização da pesquisa, o delineamento do estudo e a revisão do manuscrito.

\section{REFERÊNCIAS}

1. Organização Pan-Americana de Saúde [homepage on the internet]. Folha informativa COVID-19 [cited Jul 8 2020]. Available from: $\quad$ https://www.paho.org/bra/index. php? option $=$ com content $\&$ view $=$ article $\& i d=6101$ : covid19\&Itemid $=875$

2. Ministério da Saúde [homepage on the internet]. Sobre a doença. 0 que é COVID-19? [cited Jul 15 2020]. Available from: https://coronavirus.saude. gov.br/sobre-a-doenca

3. Ministério da Saúde [homepage on the internet]. Boletim Epidemiológico Especial: Doença pelo Coronavírus COVID-19 [cited 0ct 21 2020]. Available from: https://www.gov.br/saude/ pt-br/assuntos/boletins-epidemiologicos-1/set/ BoletimepidemiologicoCOVID33final.pdf

4. Morosini MV, Fonseca AF, Pereira IB. Educação em Saúde. In: Pereira IB, Lima JCF. Dicionário da educação profissional em saúde. 2. ed. Rio de 
Janeiro: Escola Politécnica de Saúde Joaquim Venâncio; 2008. p. 155-162.

5. Low H, Hugh B. Introdução à educação interprofissional. Fareham (UK): Centre for the Advancement of Interprofessional Education; 2013.

6. França T, Rabello ET, Magnago C. As mídias e as plataformas digitais no campo da educação permanente em saúde: debates e propostas. Saúde em Debate [serial on the internet]. 2019 [cited 0ct 21 2020];43(1):106-15. Available from: https:// www.scielo.br/pdf/sdeb/v43nspe1/0103-1104-sdeb43-spe01-0106.pdf

7. Sobral (Município). Decreto Municipal n. 2.376, de 19 de março de 2020. Intensifica as medidas para enfrentamento da infecção humana pelo novo coronavírus (COVID-19) no âmbito do município de Sobral, e dá outras providências. Sobral (CE): Secretaria Municipal de Saúde; 2020.

8. Ceccim RB. Conexões e fronteiras da interprofissionalidade: forma e formação. Interface Comun Saúde Educ [serial on the internet]. 2018 [cited 0ct 21 2020];22(Suppl 2):1739-49. Available from: https://www.scielo.br/pdf/icse/v22s2/18075762-icse-22-s2-1739.pdf

9. Peduzzi M. Educação interprofissional para o desenvolvimento de competências colaborativas em saúde. In: Toassi RFC, organizer. Interprofissionalidade e formação na saúde: onde estamos? Porto Alegre: Rede UNIDA; 2017. p. 40-48.

10. Costa MV, Peduzzi M, Freire Filho JR, Silva CBG. Educação interprofissional em saúde. Natal: Universidade Federal do Rio Grande do Norte; 2018.

11. Sousa Júnior JH, Haasch M, Soares JC, Ribeiro LVHAS. Da desinformação a caos: uma análise das fake news frente à pandemia do coronavírus (COVID-19) no Brasil. Cadernos de Prospecção [serial on the internet]. 2020 [cited 0ct 21 2020];13(2):331-46. Available from: https://cienciasmedicasbiologicas. ufba.br/index.php/nit/article/view/35978/20912

12. Brisola A, Bezerra AC. Desinformação e circulação de "fake news": distinções, diagnóstico e reação [document on the internet]. 2018 [cited Jul 10 2020]. Available from: http://hdl.handle. net $/ 20.500 .11959 / \mathrm{brapci} / 102819$

13. Kwiecinski AM, Bertagnolli SC, Villarroel MACU. Infoxicação, políticas públicas e educação. ScientiaTec [serial on the internet]. 2020 [cited Jul $102020] ; 7(1): 5-17$. Available from: file:///D:/4137Texto\%20do\%20artigo-16978-1-10-20200604.pdf

14. Silva $C P$, Rodrigues $A B$, Oliveira CLBS, Rodrigues TB, Soares NR, Dias MSA. Educação em saúde: uma revisão histórico-critica com enfoque no município

de Sobral-CE. Sanare (Sobral, Online) [serial on the internet]. 2010;9(2):29-37 [cited Nov 3 2020]. Available from: https://sanare.emnuvens.com.br/ sanare/article/view/4/2

15. Alves GG, Aerts D. As práticas educativas em saúde e a Estratégia Saúde da Família. Ciênc Saúde Colet [serial on the internet]. 2011 [cited Jul 10 2020];6(1):319-25. Available from: https://www. scielo.br/pdf/csc/v16n1/v16n1a34.pdf
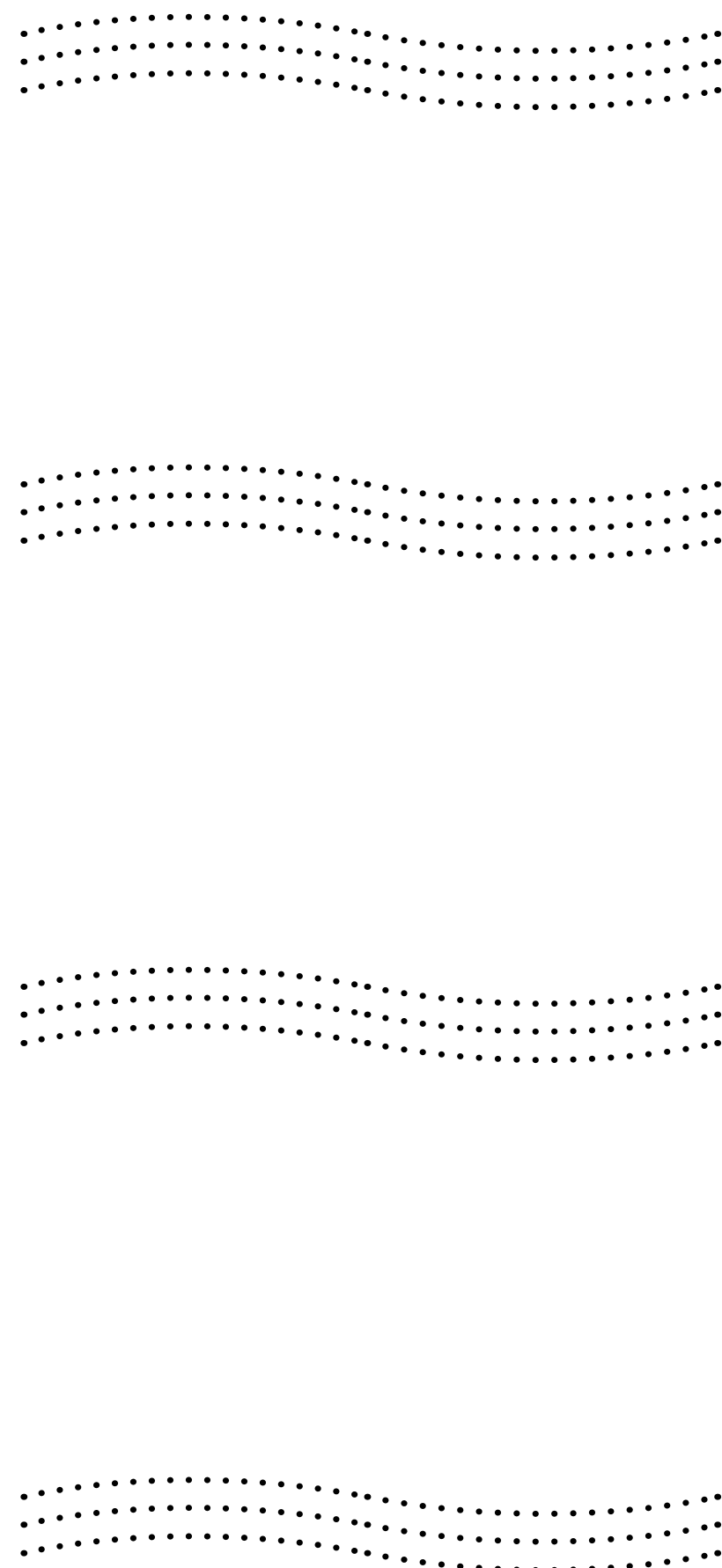